D.O.I.: $10.3895 / \mathrm{S} 1808-04482013000400006$

\title{
AVALIAÇÃO DE DESEMPENHO DE REDES DE PEQUENAS E MÉDIAS EMPRESAS (PMES): LACUNAS E OPORTUNIDADES DE PESQUISA
}

\section{PERFORMANCE EVALUATION OF SMALL AND MEDIUM ENTERPRISES (SMES) NETWORKS: GAPS AND OPPORTUNITIES RESEARCH}

\author{
Sandro César Bortoluzzi ${ }^{1}$; Sandra Rolim Ensslin ${ }^{2}$; Leonardo Ensslin ${ }^{3}$; Sandra Mara Iesbik ${ }^{4}$ \\ Valmorbida \\ ${ }^{1}$ Universidade Tecnológica Federal do Paraná - UTFPR - Pato Branco - Brasil \\ sandro@utfpr.edu.br \\ ${ }^{2}$ Universidade Federal de Santa Catarina - UFSC - Florianópolis - Brasil \\ sensslin@gmail.com \\ ${ }^{3}$ Universidade Federal de Santa Catarina - UFSC - Florianópolis - Brasil \\ leonardoensslin@gmail.com \\ ${ }^{4}$ Universidade Tecnológica Federal do Paraná - UTFPR - Francisco Beltrão - Brasil \\ smiesbik@gmail.com
}

\begin{abstract}
Resumo
Objetiva-se construir, no pesquisador, o conhecimento necessário para orientá-lo na busca de oportunidades para pesquisar sobre avaliação de desempenho de redes de PMEs. Para alcançar o objetivo utiliza-se de um processo estruturado de busca e análise de um portfólio bibliográfico nacional (33 artigos) e internacional (21 artigos). Os principais resultados são: (i) na maioria dos artigos o modelo de avaliação de desempenho foi construído em um ambiente e aplicado em outro; (ii) na maioria dos artigos os modelos são construídos sem a participação do decisor; (iii) na maioria dos artigos os autores desenvolvem o modelo para um contexto físico e o utiliza também em outros; (iv) na maioria dos modelos o processo utilizado para identificar os objetivos não leva em consideração os valores do decisor; (v) a maioria dos modelos não realizam a mensuração dos critérios; (vi) a maioria dos modelos não realiza a integração dos critérios; e, (vii) a maioria dos modelos não utiliza as informações para a gestão do desempenho. Adicionalmente, conclui-se que os artigos publicados em periódicos nacionais apresentam uma quantidade maior de lacunas comparado com os artigos publicados em periódicos internacionais.
\end{abstract}

Palavras-chave: avaliação de desempenho; redes de pequenas e médias empresas; análise sistêmica da literatura.

\section{Introdução}

A literatura apresenta que as pequenas e médias empresas (PMEs) sofrem com uma série de restrições, dentre as quais se destacam a dificuldade de inserção no mercado global (BITITCI et al, 2007; KARAEV, KOH e SZAMOSI, 2007); a ausência de planejamento estratégico (BURLAT, BESOMBES e DESLANDRES, 2003); falta de sistemas de avaliação de desempenho organizacional (CARPINETTI, GALDÁMEZ e GEROLAMO, 2008); falta de competências gerenciais e recursos, devido à ausência de trabalhadores qualificados e gestores profissionais 
(HOFFMANN e SCHLOSSER, 2001; GRANDO e BELVEDERE, 2006); falta de recursos para investimentos (HANNA e WALSH, 2008); falta de recursos e vocação para inovação (CASSANEGO JUNIOR e MAEHLER, 2010).

No entanto, a literatura também apresenta alternativas que podem ser buscadas pelas PMEs, sendo que uma dessas alternativas é a inserção em redes de cooperação (ROSENFELD, 1996; BITITCI et al, 2004; GRANDO e BELVEDERE, 2006; GEROLAMO et al, 2008; JAHN, 2009; PARRILLI, 2009; ANTONELLI e CAROLEO, 2011).

A inserção em redes de cooperação permite as empresas individuais superar diversas limitações, sendo que uma das mais relevantes é a possibilidade de evolução de seus sistemas de gestão, sendo que as ferramentas de avaliação de desempenho (AD) são frequentemente citadas como um importante instrumento de apoio ao desenvolvimento gerencial das PMEs (GARENGO; NUDURUPATI; BITITCI, 2007; GARENGO; BIAZZO; BITITCI, 2005).

A partir desses argumentos, percebe-se a importância de pesquisas científicas relacionadas a gestão de redes de PMEs, por meio da construção de ferramentas de AD.

Porém, o pesquisador antes de iniciar uma pesquisa sobre um determinado tema, precisa conhecer o que já foi publicado sobre o assunto de interesse. Esse aspecto é importante para que o pesquisador escolha temas e assuntos que apresentem potencial de contribuição ao que já foi desenvolvido pela comunidade científica. Assim, faz-se necessário buscar na literatura o que já foi publicado sobre o tema de interesse e analisar essa literatura, para que se possa identificar lacunas e oportunidades de pesquisa.

No entanto, o conhecimento acadêmico é amplamente disperso em uma variedade de publicações, bancos de dados e outras fontes de pesquisa (TASCA et al, 2010). Essa variedade e quantidade de publicações tornam a busca e a análise da literatura científica uma atividade complexa, fazendo com que os pesquisadores tenham dúvidas de como realizar uma pesquisa e análise bibliográfica em meio a tantas publicações.

Desta forma, a busca pelo conhecimento deve ser realizada por meio de um processo estruturado, que permita a seleção de um portfólio bibliográfico $(\mathrm{PB})$ relevante para o contexto do estudo a fim de fornecer um sólido quadro teórico (TASCA et al, 2010).

Cabe salientar, que construir o conhecimento necessário para iniciar uma pesquisa é singular ao pesquisador e suas delimitações, influenciado pelo ambiente onde está inserido, influenciado pelo grau de acessibilidade aos meios de divulgação de pesquisas.

Neste contexto, emerge a pergunta de pesquisa que orienta o trabalho: como construir o conhecimento demandado por um pesquisador quando do início de uma pesquisa a fim de propiciarlhe as condições necessárias para encontrar oportunidades (lacunas) em que possa contribuir em seu tema de interesse? 
Com o propósito de responder a pergunta de pesquisa, o objetivo do trabalho é construir, no pesquisador, o conhecimento necessário para orientá-lo a buscar as oportunidades (lacunas) em relação à avaliação de desempenho de redes de PMEs. Para gerar esse conhecimento, foram definidos como objetivos específicos: (i) selecionar um portfólio bibliográfico relevante sobre a gestão de redes de PMEs; (ii) identificar os critérios para realizar a análise sistêmica; e, (iii) analisar os artigos do portfólio bibliográfico quanto ao conteúdo em função dos critérios (lentes) estabelecidos (análise sistêmica da literatura).

O presente estudo justifica-se pela originalidade, importância e viabilidade (CASTRO, 1977). Originalidade por proporcionar um processo estruturado para realizar a busca de publicações científicas em periódicos nacionais e internacionais sobre o tema Avaliação de desempenho de redes de PMEs (ADRPMEs) e também por apresentar um processo para realizar a análise sistêmica das ferramentas de ADRPMEs. Com isso o estudo proporciona a identificação de lacunas e oportunidades de pesquisa em relação ao tema ADRPMEs, que poderá apoiar futuros pesquisadores sobre pesquisas inerentes à gestão de redes de PMEs por meio da AD. O estudo proporciona uma análise abrangente sobre as ferramentas de AD e proporciona diversas oportunidades de pesquisa no sentido de aperfeiçoar as ferramentas de ADRPMEs. Importante pela contribuição teórica ao tema ADRPMEs. A pesquisa apresenta viabilidade, pois as informações utilizadas são de acesso livre no portal de periódicos da Coordenação de Aperfeiçoamento de Pessoal de Nível Superior (CAPES).

O presente artigo apresenta além da introdução as seguintes seções: (i) metodologia da pesquisa; (ii) apresentação e discussão dos resultados; e, (iii) considerações finais. Por fim apresentam-se as referências utilizadas.

\section{Metodologia da pesquisa}

Essa seção está organizada da seguinte forma: (i) enquadramento metodológico; (ii) procedimentos para busca bibliográfica; e, (iii) procedimentos para coleta e análise dos dados.

\subsection{Enquadramento metodológico}

O enquadramento metodológico da pesquisa é apresentado na Figura 1: 
Figura 1 - Enquadramento metodológico da pesquisa

\begin{tabular}{|c|c|}
\hline Natureza do Objetivo: & $\begin{array}{l}\text { - Busca analisar criticamente as características de um portfólio } \\
\text { bibliográfico nacional e internacional para identificar } \\
\text { oportunidades de pesquisa sobre ADRPMEs(GIL, 1999). }\end{array}$ \\
\hline Natureza do Trabalho: & $\begin{array}{l}\text { - Busca analisar a literatura a respeito das oportunidades em } \\
\text { relação a ADRPMEs (ALAVI; CARLSON, 1992). }\end{array}$ \\
Teórico/Conceitual & $\begin{array}{c}\text { Analisou artigos publicados em periódicos nacionais e } \\
\text { internacionais sobre o tema redes de empresas (RICHARDSON, } \\
\text { 2008). }\end{array}$ \\
Coleta dos Dados: & \\
Secundários & A pesquisa não emprega ferramental estatístico na análise dos \\
Abordagem do & $\begin{array}{l}\text { dados (RICHARDSON, 2008), sendo que a análise refere-se a } \\
\text { encontrar nos artigos por meio da análise sistêmica, } \\
\text { oportunidades de pesquisa. }\end{array}$ \\
\hline Qualitativo &
\end{tabular}

Fonte: Elaborado pelos autores

Conforme enquadramento metodológico e justificativas apresentadas na Figura 1, a presente pesquisa caracteriza-se como descritiva, teórico/conceitual, a coleta de dados é secundária e a abordagem ao problema é qualitativo.

\subsection{Procedimentos para busca bibliográfica}

Para formar o portfólio bibliográfico (PB) utilizou-se o processo Knowledge Development Process- Constructivist - ProKnow-C (ENSSLIN et al, 2010).

Em relação à busca em periódicos internacionais, o processo divide-se em duas principais fases: (i) seleção do banco de artigos bruto; e, (ii) filtragem do banco de artigos.

A primeira fase é subdividida, por sua vez, em três etapas, quais sejam: (i) definir palavraschave; (ii) definir bases de dados; e, (iii) buscar artigos nas bases de dados.

Na primeira etapa definiu-se o eixo de pesquisa: $\mathrm{AD}$, com as palavras-chave: measurement, assessment, evaluation, performance e measures; o eixo PMEs, com as palavras-chave: SMEs, SME e small medium enterprise; e, o eixo redes de empresas, com as palavras-chave: cluster, district, network, cooperation, alliance e agglomeration.

A partir da definição das palavras-chave todas as possíveis combinações entre as palavraschave dos eixos de pesquisa foram formadas. Cabe salientar que a busca foi realizada por meio das 90 possíveis combinações entre as palavras-chave com o uso da expressão booleana AND que indica que o retorno de artigos acontece quando as três palavras-chave aparecem nos campos de busca. 
No desenvolvimento da segunda etapa, inicialmente, definiu-se as bases de dados para a realização da pesquisa. Como critério para seleção das bases de dados estabeleceu-se os seguintes aspectos: (i) as bases de dados deveriam constar no portal de periódicos da CAPES; (ii) para ser escolhida a base de dados deveria estar em uma das seguintes três grandes áreas da CAPES: Ciências Sociais Aplicadas, Engenharias e Multidisciplinar; (iii) as bases de dados deveriam disponibilizar ferramenta de busca com a possibilidade de uso de expressões booleanas; (iv) as ferramentas de busca das bases de dados deveriam disponibilizar a possibilidade de pesquisar nos campos: título, resumo e palavras-chave. Em função dos critérios foram identificadas e pesquisadas 23 bases de dados.

A terceira etapa consistiu em realizar a busca em cada base de dados por meio das combinações de palavras-chave. Salienta-se que os campos de busca foram: título, resumo e palavras-chave do artigo. Destaca-se também que não se restringiu a busca em relação ao período de tempo, ou seja, buscou-se o intervalo de datas disponibilizado pelas bases de dados. Esse processo resultou no banco de artigos brutos preliminar de 3.375 artigos.

A segunda fase, filtragem do banco de artigos é, subdividida, por sua vez, em seis etapas, quais sejam: (i) eliminação de artigos repetidos; (ii) alinhamento pela leitura do título; (iii) alinhamento quanto ao reconhecimento científico; (iv) alinhamento pela leitura do resumo; (v) filtro dos artigos com menor reconhecimento científico; e, (vi) alinhamento pela leitura integral dos artigos.

A primeira etapa da fase de filtragem do banco de artigos, refere-se a importação dos artigos para um software de gerenciamento bibliográfico. Desta forma, importou-se para o software os 3.375 artigos do banco de artigos bruto. Após a importação o software permite a exclusão dos artigos repetidos. Desta forma, foram excluídos 2.289 artigos repetidos e restaram 1.086 artigos não repetidos.

A segunda etapa foi a leitura dos 1.086 títulos com o objetivo de verificar o alinhamento dos artigos ao tema de pesquisa. Cabe salientar que nesta etapa buscou-se excluir os artigos que apresentavam total desalinhamento com o tema de pesquisa; na dúvida o artigo foi mantido para análise mais detalhada nas próximas etapas. Como resultado do processo de leitura restou 501 artigos alinhados ao tema de pesquisa.

A terceira etapa consiste na identificação do reconhecimento científico dos artigos. Neste processo o reconhecimento científico é aferido pelas citações em outros trabalhos; assim identificou-se a quantidade de vezes que cada artigo foi citado. Para fins de padronização buscou-se a quantidade de citações do artigo no Google acadêmico. Identificou-se 215 artigos mais citados (97\% das citações) e 286 artigos menos citados (3\% das citações). 
A quarta etapa consistiu em realizar a leitura dos 215 resumos dos artigos com maior reconhecimento científico com o objetivo de verificar o alinhamento do artigo com o tema de pesquisa. Adicionalmente aos 215 artigos foram incorporados 27 artigos das referências do portfólio bibliográfico em função do teste da representatividade do portfólio bibliográfico. Após essa etapa restaram 57 artigos que foram "guardados" no repositório $A$.

A quinta etapa teve o objetivo de filtrar os artigos com menor reconhecimento científico, ou seja, os 286 artigos com menor reconhecimento científico foram analisados para identificar aqueles que continuariam no processo. Os 17 artigos identificados nessa etapa foram "guardados" no repositório $B$. Na sequiência juntaram-se os artigos "guardados" nos repositórios $A$ (57 artigos) e $B$ (17 artigos) formando um único repositório, denominado $C$, composto por 74 artigos.

A sexta etapa da filtragem do banco de artigos consiste em fazer o alinhamento pela leitura integral do artigo. Após a eliminação dos artigos que não estavam com o texto completo disponível e leitura integral dos demais artigos, chegou-se a um portfólio bibliográfico internacional de 21 artigos científicos sobre ADRPMEs.

Em relação aos periódicos nacionais a busca ocorreu nos periódicos da área de Administração, Contabilidade e Turismo e Engenharia III, classificados com Estrato Qualis A1 à B5, pela CAPES.

O processo de seleção de artigos teve inicio com a seleção de artigos relacionada à avaliação de desempenho organizacional e na sequência selecionaram-se os artigos com foco em ADRPMEs. Foram pesquisados 408 periódicos científicos.

A segunda etapa do processo consistiu em pesquisar os 408 periódicos por meio da incidência das seguintes palavras-chave no título, resumo ou palavras-chave do artigo: (i) avaliação; (ii) desempenho; (iii) performance; (iv) decisão; (v) mensuração.

Após a realização da busca por meio das palavras-chave retornaram 22.392 artigos científicos que foram cadastrados em um software de gerenciamento bibliográfico.

$\mathrm{Na}$ etapa seguinte buscou-se o alinhamento do artigo ao tema AD pela leitura dos títulos, sendo que após a leitura dos 22.392 títulos constatou-se que apenas 5.591 títulos estavam alinhados, sendo que os demais 16.801 foram eliminados por não alinhamento.

A próxima etapa realizada foi para verificar o alinhamento do artigo ao tema AD em função da leitura dos resumos. Após a leitura dos resumos identificou-se que 668 artigos estavam alinhados e os demais artigos foram eliminados por não alinhamento.

A segunda fase do processo consistiu em verificar o alinhamento dos 668 artigos com o tema ADRPMEs. Desta forma, primeiramente realizou-se a leitura dos 668 resumos, sendo que se identificou 55 artigos alinhados ao tema. Na sequência buscou-se realizar a leitura integral dos 55 artigos para verificar os que estavam totalmente alinhados ao tema ADRPMEs. Após a leitura dos 
55 artigos, selecionou-se 33 artigos alinhados e 22 artigos foram excluídos por desalinhamento. Desta forma, 33 artigos formam o portfólio bibliográfico nacional selecionados para realizar a presente pesquisa.

\subsection{Procedimentos para análise dos dados}

A análise sistêmica integra o processo ProKnow-C, Knowledge Development ProcessConstructivist (ENSSLIN et al, 2010). A análise sistêmica é um processo científico utilizado a partir de uma visão de mundo (filiação teórica) definida e explicitada por suas lentes, analisar uma amostra de artigos representativa de um dado assunto de pesquisa, visando evidenciar para cada lente e globalmente, para a visão de mundo estabelecida, os destaques e as oportunidades (carências) de conhecimento encontrado na amostra (ENSSLIN et al, 2010).

Desta forma, cabe salientar a visão de mundo (filiação teórica) adotada para este trabalho. Avaliação de Desempenho é o processo para construir conhecimento no decisor, a respeito do contexto específico que se propõe avaliar, a partir da percepção do próprio decisor por meio de atividades que identificam, organizam, mensuram, ordinalmente e cardinalmente, integram e permitem visualizar o impacto das ações e seu gerenciamento (ENSSLIN et al, 2010).

As lentes utilizadas para analisar os artigos foram: (i) abordagem; (ii) singularidade; (iii) processo para identificar; (iv) mensuração; (v) integração; e, (vi) gestão.

Na primeira lente (abordagem) realiza-se a análise ilustrada na Figura 2:

Figura 2 - Processo utilizado para analisar a primeira lente "abordagem"

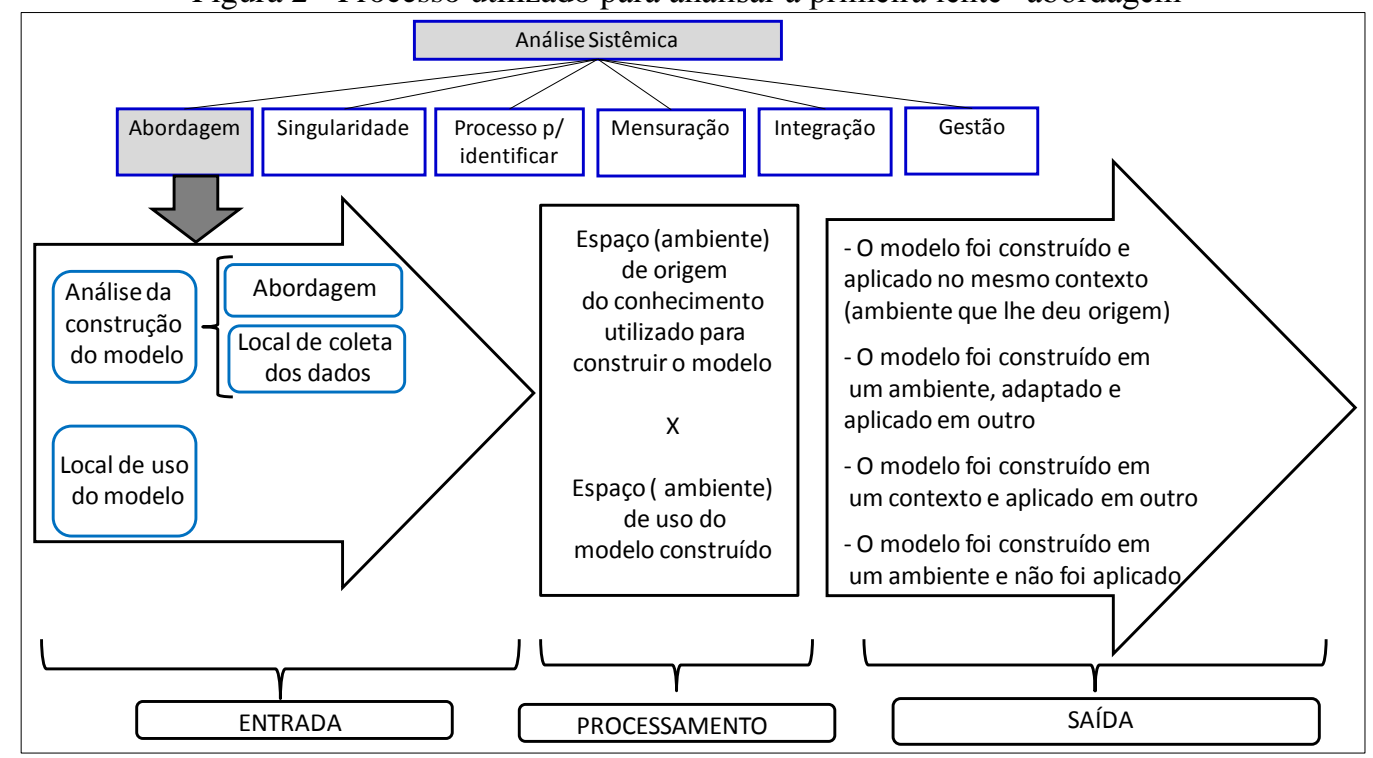

Fonte: Adaptado de Ensslin et al, (2010)

Conforme demonstrado na Figura 2 busca-se analisar a construção do modelo de AD, que se divide em abordagem e local de coleta dos dados em relação ao local de uso do modelo (entrada do processo). A análise realizada diz respeito ao confronto entre o espaço (ambiente) de origem do conhecimento utilizado para construir o modelo com o espaço (ambiente) de origem do 
conhecimento utilizado para construir o modelo (processamento). Com essa análise é possível identificar para cada artigo do PB as seguintes possíveis situações (saída): (i) o modelo foi construído e aplicado no mesmo contexto; (ii) o modelo foi construído em um ambiente, adaptado e aplicado em outro; (iii) o modelo foi construído em um contexto e aplicado em outro; e (iv) o modelo foi construído em um ambiente e não foi aplicado.

A segunda lente (singularidade) tem o propósito de analisar se os autores reconhecem que o problema é singular aos atores envolvidos no processo de $\mathrm{AD}$ e também se reconhecem que o ambiente é singular, ou seja, para cada ambiente (contexto) deve-se construir um modelo de AD (muda o contexto muda o modelo). A terceira lente (processo para identificar) tem o objetivo de identificar nos artigos analisados se os autores reconhecem os limites de conhecimentos dos decisores e também se os autores consideram os valores dos decisores na identificação dos critérios de avaliação. A quarta lente (mensuração) tem o objetivo de identificar nos artigos analisados se os autores informam o tipo de escala utilizada (nominal, ordinal, intervalo, razão), se para as escalas utilizadas os autores respeitam as propriedades de operacionalização da escala, ou seja, se os autores atendem aos princípios da homogeneidade e não ambigüidade e também se atendem a teoria da mensuração, ou seja, se em relação às operações matemáticas e estatísticas realizadas pelo artigo são compatíveis com as escalas construídas. A quinta lente (integração) tem o objetivo de identificar nos artigos analisados se os autores realizam a integração dos critérios para se ter uma avaliação global de desempenho. A sexta lente (gestão) tem o objetivo de identificar nos artigos analisados se os autores realizam o diagnóstico dos pontos fortes e fracos da situação atual e a forma pela qual realiza o diagnóstico. Outra análise realizada na lente gestão é em relação às ações de aperfeiçoamento, ou seja, se os autores geram ações para melhorar o desempenho. Neste ponto (aperfeiçoamento) busca-se analisar se o artigo gera ações de aperfeiçoamento via processo ou gera ações sem um processo estruturado e também se o artigo mensura a contribuição de cada ação na avaliação global de desempenho.

\section{Apresentação e discussão dos resultados}

A primeira análise realizada é quanto à abordagem. No Gráfico 1 apresenta-se a análise quanto á abordagem em relação aos artigos do portfólio bibliográfico. 
Gráfico 1 - Análise da lente 1 (abordagem )

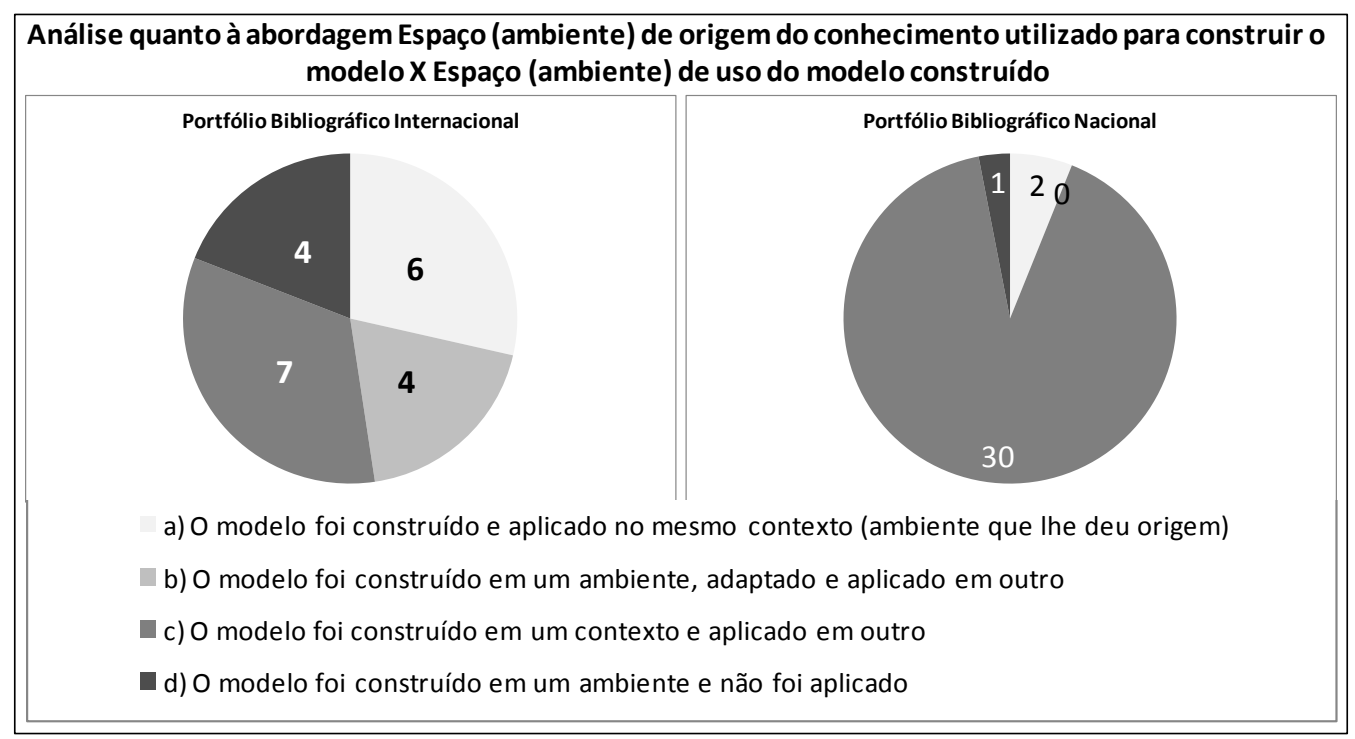

Fonte: Elaborado pelos autores

Demonstra-se que dos 21 artigos analisados do PB internacional, 6 pesquisas (29\%) o modelo foi construído e aplicado no mesmo contexto (ambiente que lhe deu origem), 4 pesquisas (19\%) o modelo foi construído em um ambiente, adaptado e aplicado em outro contexto, 7 estudos (33\%) o modelo foi construído em um contexto e aplicado em outro e 4 artigos (19\%) o modelo foi construído em um contexto e não foi aplicado.

Em relação aos 33 artigos analisados do PB nacional, 30 pesquisas (91\%) o modelo foi construído em um contexto e aplicado em outro, 2 pesquisas (6\%) o modelo foi construído em um ambiente, adaptado e aplicado em outro contexto e 1 pesquisa (3\%) o modelo foi construído e aplicado no mesmo contexto (ambiente que lhe deu origem).

Percebe-se que nos artigos publicados em periódicos internacionais, existe certo equilíbrio em relação à abordagem utilizada. No entanto, os artigos publicados em periódicos nacionais a maioria absoluta dos artigos o modelo foi construído em um ambiente e foi aplicado em outro contexto. Esse aspecto demonstra que nos artigos publicados em periódicos nacionais existe maior necessidade de aperfeiçoamento na construção dos modelos de ADRPMEs, quando analisados à luz da filiação teórica adotada para o presente trabalho.

$\mathrm{Na}$ visão de mundo adotada pelos autores, o modelo deveria ser construído e aplicado no mesmo contexto. Desta forma, percebe-se que poucos estudos da amostra analisada (nacional e internacional) têm a preocupação em construir um modelo de AD e aplicar no mesmo contexto em que foi construído. Esse aspecto (segundo a visão de mundo adotada) é uma lacuna que precisa ser preenchida na literatura sobre ADRPMEs.

A segunda lente de análise refere-se à singularidade (atores e contexto). No Gráfico 2 apresenta-se a análise quanto á singularidade (atores): 
Gráfico 2 - Análise da lente 2 (singularidade em relação aos atores)

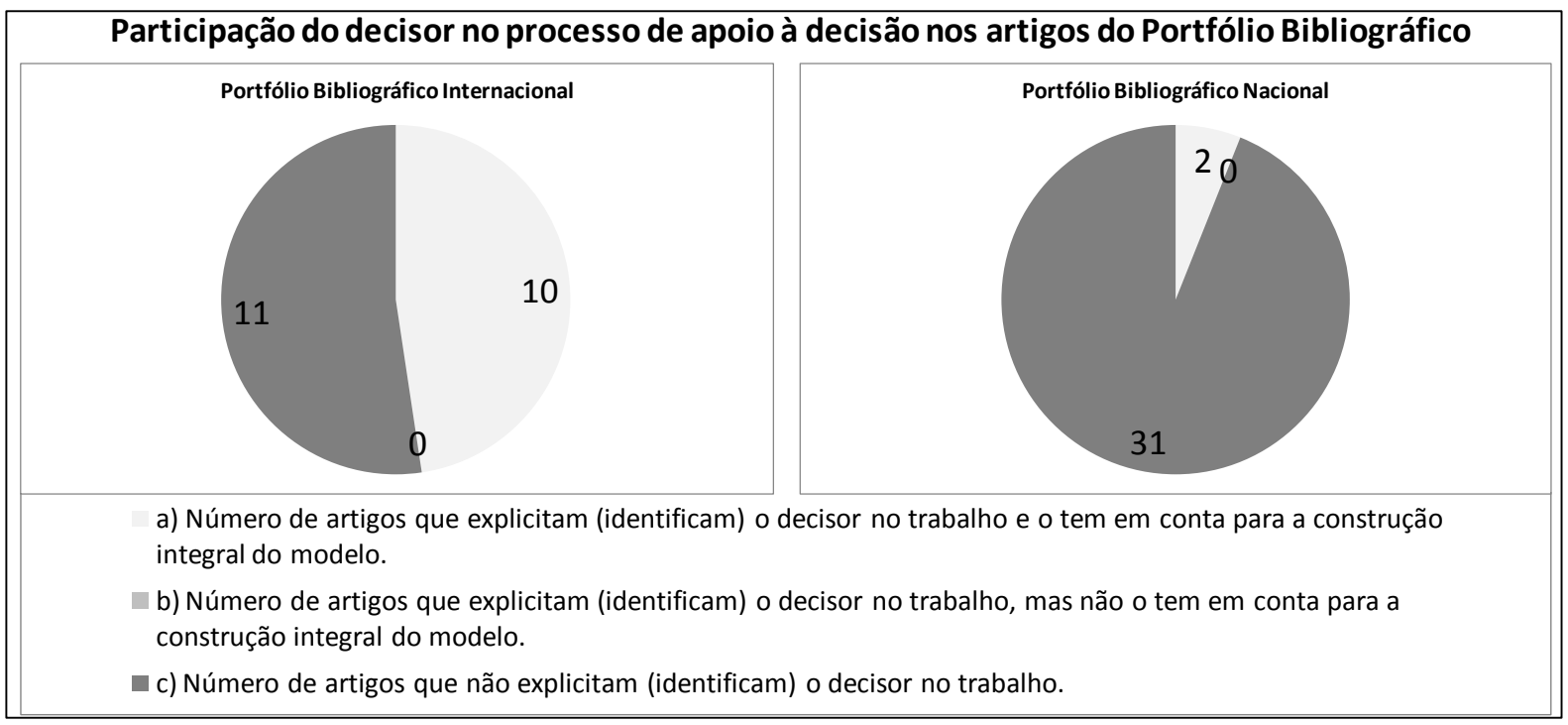

Fonte: Elaborado pelos autores

$\mathrm{Na}$ lente relativa à singularidade foi analisado se os autores reconhecem e praticam que o modelo de avaliação de desempenho deve ser construído a partir do decisor e se os mesmos identificam no trabalho quem são os decisores.

Percebe-se na análise do Gráfico 2, em relação ao PB internacional, que 10 artigos (48\%) explicitam (identificam) o decisor no trabalho e o tem em conta para a construção integral do modelo de AD e 11 artigos (52\%) não explicitam (identificam) o decisor no trabalho e também não os tem em conta na construção do modelo de AD.

Em relação aos artigos do PB nacional, demonstra-se que 2 artigos (6\%) explicitam (identificam) o decisor no trabalho e o tem em conta para a construção integral do modelo de AD e 31 artigos (94\%) não explicitam (identificam) o decisor no trabalho e também não os tem em conta na construção do modelo de $\mathrm{AD}$.

Conclui-se que existe equilíbrio entre os artigos publicados em periódicos internacionais, pois praticamente a metade dos artigos identifica e explicita os decisores e a outra metade não o faz. Porém, os artigos publicados em periódicos nacionais a maioria absoluta dos artigos não explicitam (identificam) os decisores no trabalho, o que indica a necessidade de aperfeiçoar a construção dos modelos de ADRPMEs.

Na visão de mundo adotada pelos autores, o modelo deveria ser construído considerando a singularidade dos atores, ou seja, o modelo de ADRPMEs deveria ser construído explicitando o decisor e tendo o decisor em conta na construção do modelo. Desta forma, percebe-se uma lacuna na literatura que necessita ser preenchida por futuras pesquisas.

No Gráfico 3 apresenta-se a análise quanto á singularidade (contexto): 
Gráfico 3 - Análise da lente 2 (singularidade em relação ao contexto )

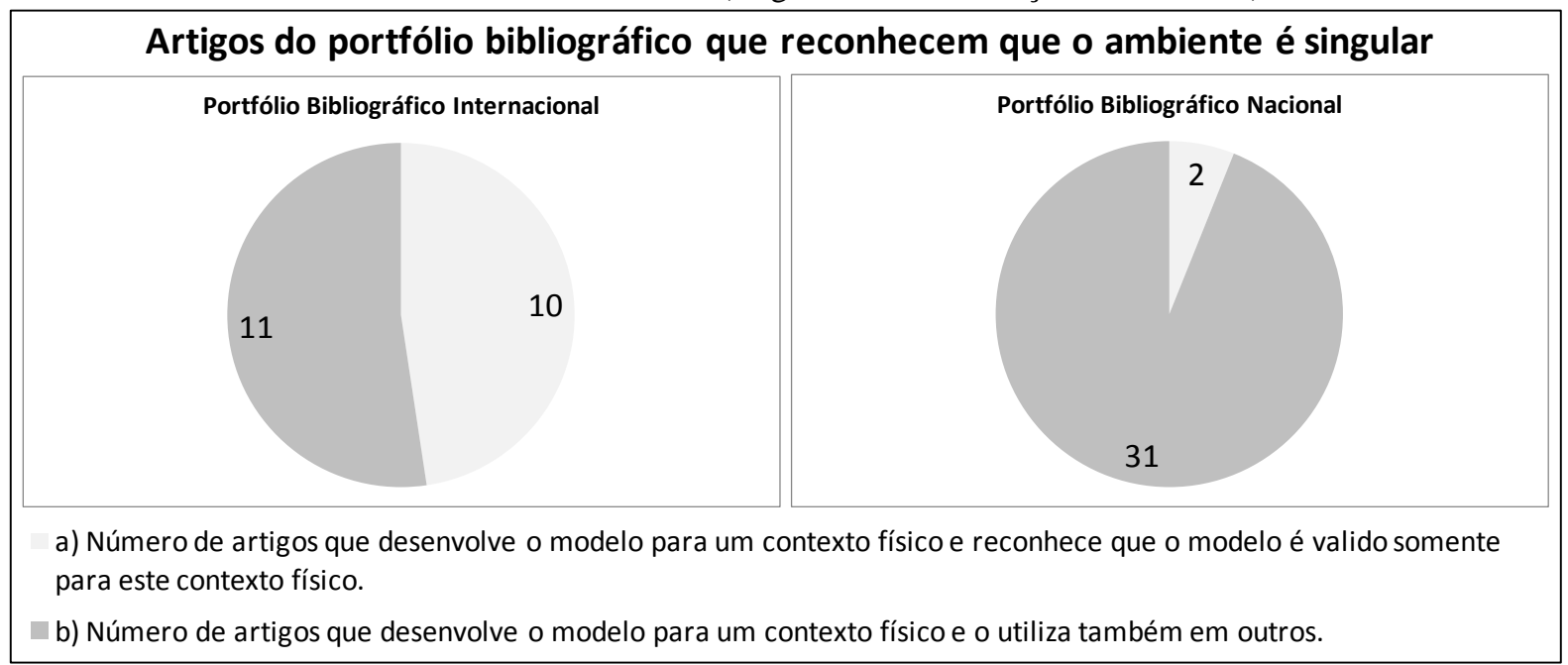

Fonte: Elaborado pelos autores

$\mathrm{Na}$ lente relativa à singularidade foi analisado também se os autores reconhecem e praticam que o modelo de $\mathrm{AD}$ deve ser construído para um determinado contexto e que se mudar o contexto outro modelo de AD deve ser construído.

Percebe-se na análise do Gráfico 3, que 10 artigos do PB internacional (48\%) desenvolvem o modelo de AD para um contexto físico e reconhecem que o modelo é válido somente para este contexto físico e 11 artigos (52\%) desenvolvem o modelo para um contexto físico e o utiliza também em outros contextos.

Em relação ao PB nacional 2 artigos (6\%) desenvolvem o modelo de AD para um contexto físico e reconhecem que o modelo é válido somente para este contexto e 31 artigos (94\%) desenvolvem o modelo para um contexto e o utiliza também em outros contextos.

Conclui-se que existe equilíbrio entre os artigos publicados em periódicos internacionais, pois praticamente a metade dos artigos é desenvolvida para um contexto e é válido somente para esse contexto. Porém, os artigos publicados em periódicos nacionais a maioria absoluta dos artigos são construídos para um contexto e aplicados também em outros, o que indica a necessidade de aperfeiçoar a construção dos modelos de ADRPMEs.

Na visão de mundo adotada pelos autores, o modelo deveria ser construído considerando a singularidade em relação ao contexto, ou seja, o modelo de ADRPMEs deveria ser construído para um contexto e aplicado somente neste contexto. Desta forma, percebe-se uma lacuna na literatura que necessita ser preenchida por futuras pesquisas.

A terceira lente de análise refere-se ao processo para identificar os critérios. Essa análise (lente) divide-se em analisar se os autores do artigo reconhecem que o decisor tem conhecimento limitado do contexto e necessita aumentar esse conhecimento e se os autores do artigo consideram os valores dos decisores na identificação dos critérios de avaliação. 
$\mathrm{Na}$ referida análise constatou-se que nenhum artigo nacional ou internacional considera importante aumentar o conhecimento do decisor sobre as variáveis que deveriam ser consideradas no modelo de AD.

$\mathrm{Na}$ visão de mundo adotada pelos autores, o modelo deveria ser construído reconhecendo que o decisor mesmo vivenciando diariamente o contexto necessita aumentar o entendimento das variáveis que deveriam ser levadas em consideração para a construção de um modelo de $\mathrm{AD}$, ou seja, o modelo de ADRPMEs deveria ser construído pela interação entre decisor e facilitador no intuito de aumentar o entendimento do que é importante levar em consideração. Desta forma, percebe-se uma lacuna a ser preenchida na literatura do tema.

No Gráfico 4 apresenta-se a análise em relação a levar em consideração os valores do decisor na construção do modelo de AD.

Gráfico 4 - Análise da lente 3 (considerar os valores do decisor)

\begin{tabular}{|l|l|}
\hline \multicolumn{2}{|c|}{$\begin{array}{c}\text { Artigos do Portfólio Bibliográfico que processo utilizado para identificar os objetivos considera os } \\
\text { valores do decisor }\end{array}$} \\
\hline \multicolumn{1}{|c|}{ Portfólio Bibligráfico Internacional } \\
$\begin{array}{l}\text { a. Número de artigos em que o processo utilizado para identificar os objetivos está integralmente alicerçado nos valores do } \\
\text { decisor; } \\
\text { b. Número de artigos em que o processo utilizado para identificar os objetivos está parcialmente alicerçado nos valores do } \\
\text { decisor; } \\
\text { c. Número de artigos em que o processo utilizado para identificar os objetivos NÃO tem em conta os valores do decisor. }\end{array}$ \\
\hline
\end{tabular}

Fonte: elaborado pelos autores

Percebe-se na análise do Gráfico 4 que 9 artigos do PB internacional (43\%) o processo utilizado para identificar os critérios está integralmente alicerçado nos valores do decisor, 1 artigo (5\%) em que o processo utilizado para identificar os critérios está parcialmente alicerçado nos valores do decisor e 11 artigos (52\%) em que o processo utilizado para identificar os critérios não tem em conta os valores do decisor.

Em relação ao PB nacional 2 artigos (6\%) o processo utilizado para identificar os critérios está integralmente alicerçado nos valores do decisor e 31 artigos (94\%) em que o processo utilizado para identificar os critérios não tem em conta os valores do decisor.

Na visão de mundo adotada pelos autores, o modelo deveria ser construído considerando os valores do decisor. Desta forma, percebe-se uma lacuna na literatura.

A quarta lente de análise refere-se a mensuração dos critérios. Primeiramente buscou-se analisar se o artigo realizava a mensuração dos critérios, apresentado no Gráfico 5: 
Gráfico 5 - Análise da lente 4 (mensuração dos critérios)

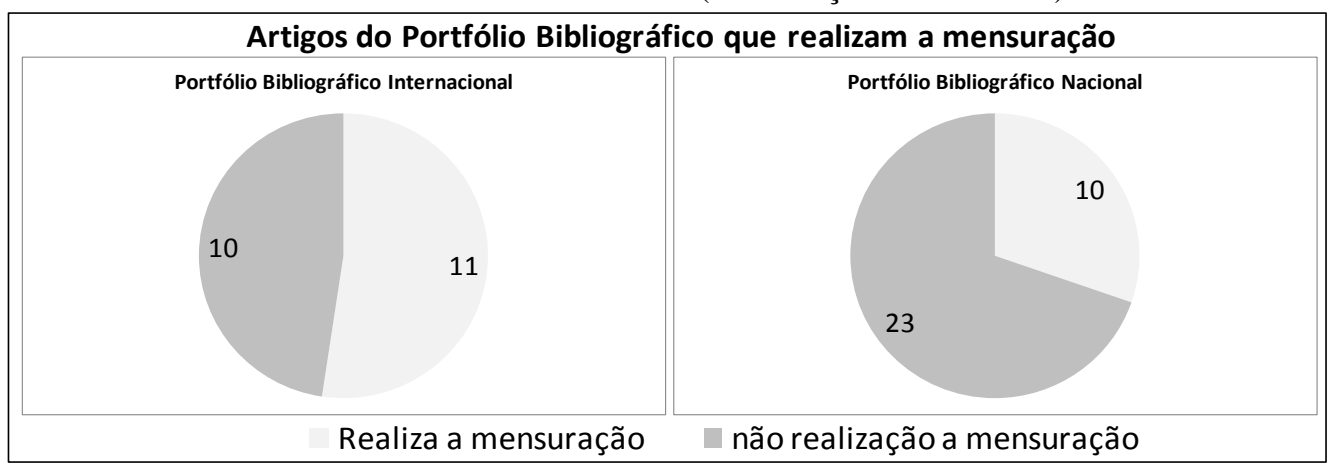

Fonte: Elaborado pelos autores

Percebe-se na análise do Gráfico 5 que 11 artigos (52\%) realizam a mensuração e 10 artigos (48\%) não realizam a mensuração em relação ao PB internacional.

Em relação ao PB nacional constata-se que 10 artigos (30\%) realizam a mensuração e 23 artigos (70\%) não realizam a mensuração.

Conclui-se que nos artigos publicados em periódicos internacionais mais da metade dos artigos realizam a mensuração. Porém, nos artigos publicados em periódicos nacionais a maioria absoluta dos artigos não realiza a mensuração dos critérios. Esse aspecto indica que, principalmente em relação aos estudos publicados em periódicos nacionais, existe a necessidade de aperfeiçoar as ferramentas e pesquisas de ADRPMEs.

Os autores do presente artigo argumentam que para a realização da $\mathrm{AD}$ ser completa, devem-se mensurar os critérios de avaliação, caso contrário a ferramenta apresentará uma lacuna considerada importante para o processo de gestão de redes de PMEs.

A segunda análise em relação à lente 4 (mensuração) foi em relação à escala utilizada. Desta forma, para os artigos que realizavam a mensuração dos critérios, buscou-se analisar se os autores do artigo informavam a escala utilizada. Nesta análise constatou-se que todos os artigos informavam a escala utilizada para mensurar os critérios. Esse aspecto demonstra que para os artigos que realizam a mensuração dos critérios os autores se preocupam em informar o tipo de escala que estão utilizando.

A terceira análise em relação à lente 4 (mensuração) foi sobre o atendimento aos princípios da homogeneidade e não ambigüidade. Desta forma, para os artigos que realizavam a mensuração dos critérios, buscou-se analisar se os autores do artigo atendiam a esses dois princípios. O resultado dessa análise é apresentado no Gráfico 6: 
Gráfico 6 - Análise da lente 4 (mensuração dos critérios - princípios)

\begin{tabular}{|c|c|}
\hline \begin{tabular}{c} 
Artigos do portfólio bibliográfico que as escalas atendem as propriedades da: \\
homogeneidade; não ambiguidade \\
\hline Portfólio bibliográfico Internacional \\
\hline 11
\end{tabular} \\
\hline \\
Número de artigos em que as escalas utilizadas atendem aos princípio da: homogeneidade; não ambiguidade \\
Número de artigos em que as escalas utilizadas não atendem aos princípio da: homogeneidade; não ambiguidade \\
\hline
\end{tabular}

Fonte: Elaborado pelos autores

Percebe-se na análise do Gráfico 6 que todos os artigos do PB internacional que realizam a mensuração dos critérios atendem aos princípios da homogeneidade e não ambigüidade. Em relação ao PB nacional constata-se que 8 artigos (73\%) atendem aos princípios da homogeneidade e não ambigüidade e que 3 artigos (27\%) não atendem.

A quarta análise refere-se a lente 4 (mensuração) que buscou analisar as operações matemáticas e estatísticas realizadas para as escalas construídas. Desta forma, para os artigos que realizavam a mensuração dos critérios, buscou-se analisar se os autores do artigo atendiam a teoria da mensuração. O resultado dessa análise é apresentado no Gráfico 7:

Gráfico 7 - Análise da lente 4 (mensuração dos critérios - teoria da mensuração)

\begin{tabular}{|l|l|}
\hline \begin{tabular}{|l|}
\hline Artigos do portfólio bibliográfico em que as escalas atendem as propriedades da teoria \\
da mensuração
\end{tabular} \\
\hline 4 \\
\hline APortfólio Bibliográfico Internacional \\
Número de artigos em que as operações matemáticas e estatísticas realizadas são compatíveis com as escalas construídas. \\
Número de artigos em que as operações matemáticas e estatísticas realizadas não são compatíveis com as escalas construídas. \\
\hline
\end{tabular}

Fonte: Elaborado pelos autores

Percebe-se na análise do Gráfico 7, que 7 artigos do PB internacional (64\%) as operações matemáticas e estatísticas realizadas são compatíveis com as escalas construídas e que 4 artigos $(36 \%)$ as operações matemáticas e estatísticas realizadas não são compatíveis com as escalas construídas. 
Em relação ao PB nacional 8 artigos (73\%) as operações matemáticas e estatísticas realizadas são compatíveis com as escalas construídas e que 3 artigos (27\%) as operações matemáticas e estatísticas realizadas não são compatíveis com as escalas construídas.

A quinta lente de análise refere-se à integração dos critérios. Primeiramente buscou-se analisar se o artigo realizava a integração dos critérios, conforme apresentado no Gráfico 8.

Gráfico 8 - Análise da lente 5 (integração dos critérios)

\begin{tabular}{|c|c|}
\hline \multicolumn{2}{|c|}{ Artigos do portfólio bibliográfico que realizam a integração dos indicadores } \\
\hline 20 & \\
\hline Portfólio Bibliográfico Internacional & 31 \\
\hline Realiza a integração & Não realiza a integração \\
\hline
\end{tabular}

Fonte: Elaborado pelos autores

Percebe-se na análise do Gráfico 8, que apenas um artigo do PB internacional preocupa-se com a integração dos critérios para se ter uma avaliação global de desempenho.

Em relação ao PB nacional constata-se que apenas dois artigos preocupam-se com a integração dos critérios para se ter uma avaliação global de desempenho. Esse aspecto evidencia uma lacuna importante na ADRPMEs.

Após analisar a quantidade de artigos que realizavam a integração, buscou-se identificar a forma que a integração foi realizada. Desta forma, constatou-se que o artigo do PB internacional que realiza a integração o faz por meio do estabelecimento de níveis de referência, sendo que essa forma de realizar a integração está alinhada com a filiação teórica dos autores do presente estudo. Em relação ao $\mathrm{PB}$ nacional, os dois artigos que realizam a integração o fazem sem o estabelecimento de níveis de referência, ou seja, desalinhado com a filiação teórica do presente estudo.

A sexta lente de análise refere-se à gestão do desempenho. Essa análise está dividida em diagnóstico e aperfeiçoamento. Primeiramente buscou-se analisar se o estudo permitia realizar o diagnóstico dos pontos fortes e fracos da situação atual, conforme Gráfico 9: 
Gráfico 9 - Análise da lente 6 (gestão - diagnóstico)

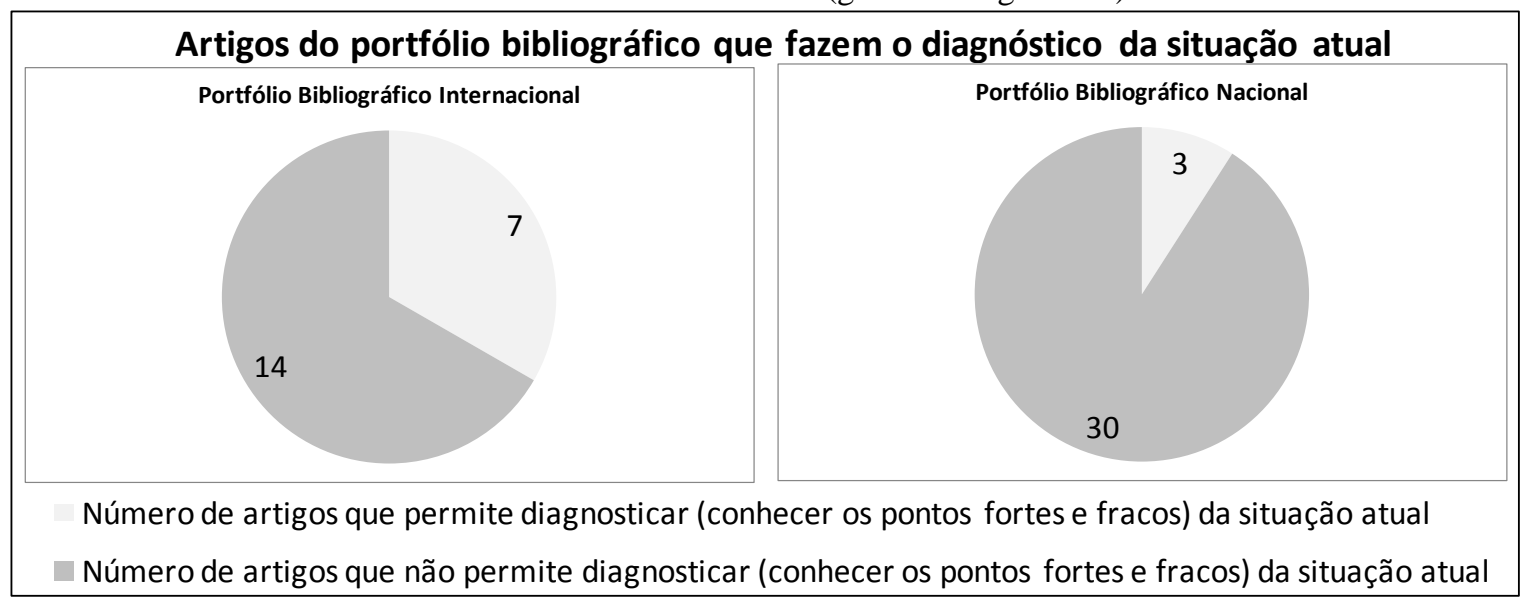

Fonte: Elaborado pelos autores

Percebe-se na análise do Gráfico 9 que 7 artigos do PB internacional (33\%) permitem diagnosticar (conhecer os pontos fortes e fracos) da situação atual e que 14 artigos (67\%) não permite fazer esse diagnóstico. Em relação ao PB nacional, constata-se que 3 artigos (9\%) permitem diagnosticar (conhecer os pontos fortes e fracos) da situação atual e que 30 artigos (91\%) não permitem fazer esse diagnóstico. Esse aspecto é visto como importante para o processo de AD segundo a filiação teórica adotada. Desta forma, percebe-se uma lacuna na literatura e uma oportunidade para a presente pesquisa.

A segunda análise em relação à gestão foi a identificação da forma pela qual os artigos que realizavam o diagnóstico o faziam. Constatou-se nesta análise que todos os artigos (portfólio bibliográfico nacional e internacional) que realizam o diagnóstico o fazem de uma forma descritiva.

A próxima análise em relação à gestão do desempenho diz respeito ao aperfeiçoamento, ou geração de ações de aperfeiçoamento do desempenho. Primeiramente buscou-se analisar a quantidade de artigos que geravam ações de aperfeiçoamento. Nesta análise constatou-se que nenhum artigo do portfólio bibliográfico nacional e internacional analisado disponibilizam processo para gerar ações de aperfeiçoamento. Segundo a filiação teórica adotada esse aspecto é importante no processo de ADRPMEs. Esse aspecto evidencia uma lacuna na literatura e uma oportunidade para o presente estudo.

\section{Considerações finais}

O presente estudo procurou responder a seguinte pergunta de pesquisa: como construir o conhecimento demandado por um pesquisador quando do início de uma pesquisa a fim de propiciarlhe as condições necessárias para encontrar oportunidades (lacunas) em que possa contribuir em seu tema de interesse? Com o propósito de responder a pergunta de pesquisa, o objetivo do trabalho foi construir, no pesquisador, o conhecimento necessário para orientá-lo a buscar as oportunidades (lacunas) em relação à avaliação de desempenho de redes de PMEs. A pergunta de pesquisa e o 
objetivo geral foram respondidos, pois se conseguiu por meio de um processo estruturado de busca identificar um PB nacional e internacional relevante e alinhado ao tema ADRPMEs. Adicionalmente, o PB (nacional e internacional) foi analisado em função de seis lentes identificadas por meio da filiação teórica dos autores em relação à $\mathrm{AD}$. A busca bibliográfica realizada e a análise sistêmica permitiram identificar oportunidades de pesquisa em relação aos estudos de ADRPMEs.

O primeiro objetivo específico foi selecionar um PB relevante sobre a gestão de redes de PMEs, sendo que o mesmo foi alcançado por meio de um processo estruturado de busca que permitiu identificar 21 artigos publicados em periódicos internacionais e 33 artigos publicados em periódicos nacionais sobre o tema ADRPMEs, conforme demonstrado na seção 2.2 - procedimentos para busca bibliográfica.

O segundo objetivo específico foi identificar os critérios para realizar a análise sistêmica, sendo que o mesmo foi alcançado por meio da definição de seis lentes de análise (abordagem, singularidade, processo para identificar, mensuração, integração e gestão). Essas lentes foram extraídas da filiação teórica adotada pelos autores, sendo que essas lentes representam o entendimento dos pesquisadores do presente estudo do que é avaliar o desempenho organizacional. Cabe salientar que a definição das lentes foi realizada na seção 2.3 - procedimentos para a análise dos dados.

O terceiro objetivo específico foi analisar os artigos do portfólio bibliográfico quanto ao conteúdo em função dos critérios (lentes) estabelecidos (análise sistêmica da literatura). Após a seleção do PB e a definição dos critérios (lentes) de análise, procedeu-se a análise dos artigos. A análise foi realizada pela leitura dos artigos do PB e a confrontação desses estudos com a filiação teórica (lentes).

Os principais resultados da análise sistêmica foram: (i) em relação à abordagem utilizada pelos autores percebe-se que a maioria dos estudos o modelo de avaliação de desempenho é construído em um contexto e aplicado em outro contexto; (ii) em relação à singularidade percebe-se que a maioria dos estudos não se preocupa em explicitar (identificar) os atores que participam do processo de construção do modelo de avaliação de desempenho e também a maioria dos artigos não levam em consideração o contexto específico para construção do modelo, o que caracteriza que os modelos construídos para avaliar o desempenho de redes de PMEs são modelos genéricos, utilizados em outros contextos, e não especificamente para avaliar uma rede de empresas particular; (iii) em relação à identificação dos critérios constata-se que nenhum artigo preocupa-se em construir conhecimento no decisor, ou seja, o modelo é construído por meio da geração de conhecimento no facilitador e/ou pela busca de informações na literatura, entre outros meios. Adicionalmente, constatou-se que a maioria dos estudos não leva em consideração o decisor para identificação dos objetivos a serem avaliados; (iv) em relação à mensuração concluiu-se que a 
maioria dos estudos não realiza a mensuração dos critérios, sendo que aqueles que o fazem informam o tipo de escala que utilizam para realizar a mensuração. Adicionalmente, observou-se que a maioria dos artigos atende aos princípios da homogeneidade e não ambigüidade e que a maioria dos artigos atendem ao princípio da mensuração, pois utilizam operações matemáticas e estatísticas compatíveis com as escalas construídas; (v) em relação à integração dos critérios percebe-se que a maioria absoluta dos artigos não realiza a integração dos critérios para se ter uma avaliação global de desempenho; (vi) em relação à gestão, concluiu-se que a maioria dos estudos não permite identificar os pontos fortes e fracos da situação atual e também que nenhum artigo apresenta processo para gerar ações de aperfeiçoamento do desempenho.

Na comparação da literatura internacional em relação à nacional percebe-se que os artigos publicados em periódicos nacionais têm um caminho mais longo a percorrer, quando analisados à luz da filiação teórica adotado no presente estudo.

Dessa forma, conclui-se que a ADRPMEs é um tema que apresenta uma série de lacunas que precisam ser preenchidas por novos estudos.

A presente pesquisa apresenta as seguintes limitações: (i) o processo de busca realizado considerou apenas os periódicos disponibilizados no portal da CAPES; (ii) deixa de realizar uma proposta de ferramenta que atenda as lacunas identificadas. Dessa forma, sugere-se para futuras pesquisas, ampliar a busca em outros periódicos e incluir também anais de eventos e desenvolver uma ferramenta de avaliação de desempenho para redes de PMEs que atenda as lacunas identificadas na literatura.

\section{Abstract}

It aims to build on researcher, the knowledge to guide you in finding opportunities to research on performance evaluation of SMEs networks. To achieve the objective uses a structured process of searching and analyzing a national bibliographic portfolio (33 articles) and international (21 articles). The results present gaps in relation to the form of identification, measurement, integration of the criteria and the absence of a process for the management and improvement of SMEs networks. Additionally, we conclude that the articles published in national journals have a larger number of gaps compared with articles published in international journals.

Key-words: performance evaluation; small and medium enterprise network; systemic analysis of the literature.

\section{Referências}

ALAVI, M.; CARLSON, P. A review of MIS research and disciplinary development. Journal of Management Information Systems, v. 8, n. 4, p. 45-62, 1992.

ANTONELLI, D.; CAROLEO, B. An integrated methodology for the analysis of collaboration in industry networks. Journal of Intelligent Manufacturing, in press.

BITITCI, U. S.; MARTINEZ, V.; ALBORES, P.; PARUNG, J. Creating and managing value in collaborative networks. International Journal of Physical Distribution \& Logistics Management, v. 34, n. 3, p. 251-268, 2004. crossref 
BITITCI, U. S.; TURNER, T.; MACKAY, D.; KEARNEY, D.; PARUNG, J. WALTERS, D. Managing synergy in collaborative enterprises. Production Planning \& Control, v. 18, n. 6, p. 454-465, 2007. cross ref

BURLAT, P.; BESOMBES, B.; DESLANDRES, V. Constructing a typology for networks of firms. Production Planning \& Control, v. 14, n. 5, p. 399-409, 2003. crossref

CARPINETTI, L. C. R.; GALDÁMEZ, E. V. C.; GEROLAMO, M. C. A measurement system for managing performance of industrial clusters: A conceptual model and research cases. International Journal of Productivity and Performance Management, v. 57, n. 5, p. 405-419, 2008. cross ref

CASSANEGO JR, P; MAEHLER, A. E. O programa de apoio aos arranjos produtivos locais (APL'S) no RS: Uma política de criação de vantagem competitiva as empresas de micro e pequeno porte. RDE Revista de Desenvolvimento Econômico, v. 12, n. 21, p. 62-73, 2010.

CASTRO, C. M. A prática da pesquisa. São Paulo: McGraw-Hill do Brasil, 1977.

ENSSLIN, L.; ENSSLIN, S. R.; LACERDA, R. T. O.; TASCA, J. E.. ProKnow-C, Knowledge Development ProcessConstructivist. Processo técnico com patente de registro pendente junto ao INPI. Brasil, 2010.

GARENGO, P.; BIAZZO, S.; BITITCI, U. S. Performance measurement systems in SMEs: A review for a research agenda. International Journal of Management Reviews, v. 7, n. 1, p. 25 47, Mar 2005.

GARENGO, P.; NUDURUPATI, S.; BITITCI, U. Understanding the relationship between PMS and MIS in SMEs: an organizational life cycle perspective. Computers in Industry, v. 58, n. 7, p. 677-686, 2007. crossref

GEROLAMO, M. C.; CARPINETTI, L. C. R.; SELIGER, G.; GALDÁMEZ, E. V. C. Performance management of regional clusters and SME cooperation networks. International Journal of Business Excellence, v. 1, n. 4, p. 457-483, 2008. crossref

GIL, A. C. Como elaborar projetos de pesquisa. $3^{\circ}$ Edição. São Paulo: Atlas, 1999.

GRANDO, A.; BELVEDERE, V. District's manufacturing performances: A comparison among large, small-tomedium-sized and district enterprises. International Journal of Production Economics, v. 104, n. 1, p. 85-99, 2006. crossref

HANNA, V.; WALSH, K. Interfirm cooperation among small manufacturing firms. International Small Business Journal, v. 26, n. 3, p. 299-321, 2008. crossref

HOFFMANN, W. H.; SCHLOSSER, R. Success factors of strategic alliances in small and medium-sized enterprises an empirical survey. Long Range Planning, v. 34, n. 3, p. 357-381, 2001. crossref

JÄHN, H. Value-added process-related performance analysis of enterprises acting in cooperative production structures. Production Planning \& Control, v. 20, n. 2, p. 178-190, 2009. crossref

KARAEV, A.; KOH, S. C. L.; SZAMOSI, L. T. The cluster approach and SME competitiveness: A review. Journal of Manufacturing Technology Management, v. 18, n. 7, p. 818-835, 2007. crossref

PARRILLI, M. D. Collective efficiency, policy inducement and social embeddedness: Drivers for the development of industrial districts. Entrepreneurship \& Regional Development, v. 21, n. 1, p. 1-24, 2009. crossref

RICHARDSON, R. J. Pesquisa Social: Métodos e Técnicas. $3^{\circ}$ Edição. São Paulo: Atlas, 2008.

ROSENFELD, S. A. Does cooperation enhance competitiveness? Assessing the impacts of inter-firm collaboration. Research Policy, v. 25, edição especial, p. 247-263, 1996.

TASCA, J. E.; ENSSLIN, L.; ENSSLIN, S. R.; ALVES, M. An approach for selecting a theoretical framework for the evaluation of training programs. Journal of European Industrial Training, v. 34, n. 7, p. 631-655, 2010. crossref 


\section{Dados dos autores}

Nome completo: Sandro César Bortoluzzi

Filiação institucional: Universidade Tecnológica Federal do Paraná

Departamento: Ciências Contábeis

Função ou cargo ocupado: Professor

Endereço completo para correspondência (bairro, cidade, estado, país e CEP):

Rua Itapuã, 875 - Apto 202 - Bairro: Parzianelo, Pato Branco, Paraná, Brasil e CEP: 85.504-060.

Telefones para contato: 46-3220-2527

e-mail: sandro@utfpr.edu.br

Nome completo: Sandra Rolim Ensslin

Filiação institucional: Universidade Federal de Santa Catarina

Departamento: Ciências Contábeis

Função ou cargo ocupado: Professora

Endereço completo para correspondência (bairro, cidade, estado, país e CEP):

Rua Itararé, 41, Parque São Jorge, Itacorubi - Florianópolis - Santa Catarina -

CEP: 88.034-470.

Telefones para contato: 48-3721-6608

e-mail: sensslin@gmail.com

Nome completo: Leonardo Ensslin

Filiação institucional: Universidade Federal de Santa Catarina

Departamento: Engenharia de Produção

Função ou cargo ocupado: Professor

Endereço completo para correspondência (bairro, cidade, estado, país e CEP):

Endereço: Rua Frei Caneca, 629, apt. 402, Centro - Florianópolis - Santa Catarina -

CEP: $88.025-000$

Telefones para contato: 48-3721-6608

e-mail: leonardoensslin@gmail.com

\section{Nome completo: Sandra Mara Iesbik Valmorbida}

Filiação institucional: Universidade Tecnológica Federal do Paraná

Departamento: Gestão e Planejamento

Função ou cargo ocupado: Técnico Administrativo

Endereço completo para correspondência (bairro, cidade, estado, país e CEP): 
Linha Santa Bárbara, s/n, Francisco Beltrão, Paraná, Brasil e CEP: 85.601-970

Telefones para contato: 46-3523-6370

e-mail:smiesbik@gmail.com

Submetido em: 05/12/2012

Aceito em: 10/12/2013 